\title{
Estimation of the risk of secondary malignancy arising from whole-breast irradiation: comparison of five radiotherapy modalities, including TomoHDA
}

\author{
Eun Young Han ${ }^{1}$, Nava Paudel ${ }^{2}$, Jiwon Sung ${ }^{3}$, Myonggeun Yoon ${ }^{3}$, Weon Kuu Chung ${ }^{4}$ \\ and Dong Wook Kim ${ }^{4}$ \\ ${ }^{1}$ Department of Radiation Physics, The University of Texas MD Anderson Cancer Center, Houston, TX, USA \\ 2 Department of Radiation Oncology, University of Arkansas for Medical Sciences, Little Rock, AR, USA \\ ${ }^{3}$ Department of Bio-Convergence Engineering, Korea University, Seoul, Korea \\ ${ }^{4}$ Department of Radiation Oncology, Kyung Hee University Hospital at Gangdong, Seoul, Korea \\ Correspondence to: Dong Wook Kim, email: joocheck@gmail.com
}

Keywords: secondary malignancy, breast, IMRT, VMAT, TomoHDA

Received: November 05, 2015 Accepted: March 18, 2016

Published: March 26, 2016

\section{ABSTRACT}

The risk of secondary cancer from radiation treatment remains a concern for long-term breast cancer survivors, especially those treated with radiation at the age younger than $\mathbf{4 5}$ years. Treatment modalities optimally maximize the dose delivery to the tumor while minimizing radiation doses to neighboring organs, which can lead to secondary cancers. A new TomoTherapy treatment machine, TomoHDA ${ }^{\mathrm{TM}}$, can treat an entire breast with two static but intensity-modulated beams in a slice-byslice fashion. This feature could reduce scattered and leakage radiation doses. We compared the plan quality and lifetime attributable risk (LAR) of a second malignancy among five treatment modalities: three-dimensional conformal radiation therapy, field-in-field forward-planned intensity-modulated radiation therapy, inverse-planned intensity-modulated radiation therapy (IMRT), volumetric modulated arc therapy, and TomoDirect mode on the TomoHDA system. Ten breast cancer patients were selected for retrospective analysis. Organ equivalent doses, plan characteristics, and LARs were compared. Out-of-field organ doses were measured with radiophotoluminescence glass dosimeters. Although the IMRT plan provided overall better plan quality, including the lowest probability of pneumonitis, it caused the second highest LAR. The TomoTherapy plan provided plan quality comparable to the IMRT plan and posed the lowest total LAR to neighboring organs. Therefore, it can be a better treatment modality for younger patients who have a longer life expectancy.

\section{INTRODUCTION}

Evolving early-stage breast cancer treatment strategies have improved the survival of patients who undergo breast conservation surgery. After surgery, patients generally receive radiation treatment of the entire breast with a dose of 50.4 Gy [1]. This strategy reduces local cancer recurrence by one half to two thirds and the chance of death due to breast cancer by about one sixth [2].

However, among $>10$-year survivors, the probability of developing a secondary malignancy increases significantly in women who receive the radiation treatment at the age of 45 years or younger [3]. To improve these patients' long-term survival and quality of life, minimizing scattered and leakage radiation dose to normal tissues or organs while maintaining tumor control becomes more critical [4].

Various treatment modalities have been applied for breast cancer treatment using a traditional linear accelerator (Linac). Patients can be treated by threedimensional conformal radiation therapy (3D-CRT), fieldin-field forward-planned intensity-modulated radiation therapy (FinF), standard intensity-modulated radiation therapy (IMRT), and volumetric modulated arc therapy (VMAT). The newly introduced TomoHDA ${ }^{\mathrm{TM}}$ machine (v2.0, Accuray, Madison, WI, USA) can deliver IMRT beams at static angles in a slice-by-slice fashion while 
Table 1: Patient characteristics

\begin{tabular}{|l|l|l|l|}
\hline Patient number & Breast site & Tangential beam angles $(\mathbf{o})$ & PTV volume $\left.\mathbf{( c m}^{\mathbf{3}}\right)$ \\
\hline 1 & Left & $317.4 / 127.2$ & 1421 \\
\hline 2 & Left & $323.6 / 129$ & 733 \\
\hline 3 & Left & $305.9 / 118.8$ & 596 \\
\hline 4 & Left & $319.7 / 127.8$ & 291 \\
\hline 5 & Left & $315.7 / 122.2$ & 726 \\
\hline 6 & Right & $239.3 / 49.1$ & 485 \\
\hline 7 & Right & $235.0 / 38.1$ & 1025 \\
\hline 8 & Right & $233.5 / 35$ & 866 \\
\hline 9 & Right & $243.6 / 50$ & 904 \\
\hline 10 & Right & $233.1 / 41$ & 381 \\
\hline
\end{tabular}

a patient slowly moves into the beam. This treatment modality is called TomoDirect ${ }^{\mathrm{TM}}$ (TOMO). Another advanced feature of the TomoHDA system is that two field-defining jaws move dynamically to conform to a target in the craniocaudal direction, which can produce a sharp dose fall-off.

Previous comparative studies have shown that the risk of secondary cancer induction is higher with an IMRT plan than with a 3D-CRT plan. This is attributed to increased out-of-field leakage radiation due to the higher number of fields and monitor units (MUs) used in the IMRT plan. But the plan quality of the IMRT plan, with regard to factors such as planning target volume (PTV) dose coverage and organ-at-risk (OAR) dose reductions, is generally better than that of the 3D-CRT plan [5-9]. In a comparative study of oropharyngeal cancer treatment plans, Gestel et al. reported that the helical TomoTherapy plan elicited better homogeneous PTV dose coverage and better OAR sparing than other modalities. However, the lifetime attributable risks (LARs) between treatment modalities were not compared [10]. The purpose of the study described here is to compare the plan quality and LAR among five treatment modalities (3D-CRT, FinF, IMRT, VMAT, and TOMO). Our hypothesis is that the TomoDirect modality (TOMO) can maintain the plan quality of the Linac-based IMRT plan and still lower LAR compared with the other treatment modalities.

\section{MATERIALS AND METHODS}

\section{Patient selection and planning parameters}

Ten female patients with breast cancer $(5$ left sided and 5 right sided) were retrospectively selected for this study. As shown in Table 1, PTVs among the ten patients ranged from 291 to $1421 \mathrm{~cm}^{3}$.

A CT simulator (Brilliance CT, Philips Medical System, Netherlands) was used to obtain planning CT images with $3 \mathrm{~mm}$ slice thickness for patients in supine position with both arms up with an aid of a wing board.
TOMO plans for all patients were optimized with the TomoTherapy (v 5.0) planning system, and the Linacbased (21iX Varian Medical System, Palo Alto, CA) plans were optimized with the Eclipse treatment planning system (v 8.9, Varian Medical System, Palo Alto, CA).

PTV was defined as the "ipsilateral" breast (the breast containing the tumor) with $5 \mathrm{~mm}$ skin extraction. The lungs, heart, and contralateral breast were contoured as OARs. The prescription dose was set to $50.4 \mathrm{~Gy}$ in 28 fractions, and the plan was normalized as this dose covering $95 \%$ of the PTV. Dose constraints for ipsilateral lung volumes receiving $20 \mathrm{~Gy}$ and $10 \mathrm{~Gy}$ doses (V20 and $\left.\mathrm{V} 10_{\mathrm{Gy}}\right)$ were set as less than $20 \%$ and $40 \%$ of the lung volume, respectively. The maximum spinal cord dose was limited to less than 45 Gy [11]. Doses to the other organs were kept as low as possible.

For each patient, five different plans were created using the five treatment modalities. Figure 1 shows a graphical view of dose distributions and beam arrangements of the treatment plans. The 3D-CRT plan consisted two parallel opposed tangential beams. The FinF plan has the same gantry angles as the 3D-CRT plan but added sub-fields created by a multi-leaf collimator (MLC) for dose compensation. The IMRT plan consisted as IMRT field can consist any number of beams $\sim>5$ of 10 to 12 fields to spare the contralateral breast, lung, and heart. Recently, Li et al. reported a non-isocentric IMRT treatment strategy for breast radiation therapy [12], with significant reduction of ipsilateral lung and heart doses; however, this technique takes longer planning and treatment time, instead we generated a single isocentric IMRT plan and increased the number of fields to ensure PTV dose coverage. The range of the gantry angles of the IMRT plans was relatively larger than that of the 3D-CRT plan by $\sim 26.5^{\circ} \pm 9.8^{\circ}$. The VMAT plan consisted of 3 to 4 partial arcs covering a range of beam angles similar to that of the IMRT plan. For the left breast irradiation, the average gantry angle spanned from $305^{\circ}$ to $152^{\circ}$, and for the right breast irradiation the average gantry angle spanned from $60^{\circ}$ to $214^{\circ}$. For the TOMO plan, a field width of $5.0 \mathrm{~cm}$, a pitch of 0.4 , and a maximum modulation factor of 2.067 were used. 
In this study, contralateral breast, contralateral and ipsilateral lung, and "contralateral" and "ipsilateral" heart (corresponding to right-sided and left-sided breast cancers, respectively) were considered in-field organs. Six out-of-field organs - thyroid, stomach, liver, colon, gonad, and rectum - were selected and analyzed. Doses to in-field organs were derived from dose-volume histograms (DVHs), and doses to out-of-field organs were derived from measurements using a radio-photoluminescence glass dosimeter (RPLGD).

\section{Out-of-field organ dose measurement using a radio-photoluminescence glass dosimeter}

Out-of-field dosimetry requires a direct measurement as commercial treatment planning systems cannot estimate dose outside the radiation field well $[13,14]$. We used RPLGD dosimeters (A.T.G., Chiyoda Technology Corporation, Tokyo, Japan) to estimate the out-of-field organ doses. RPLGD has good reproducibility and low energy dependency at energy levels $>200 \mathrm{keV}$ $[15,16]$. Small differences in individual sensitivity, repeatable readout, and excellent accuracy and stability at room temperature make RPLGD suitable for the dosimetry of scattered radiation outside the treatment field [17, 18]. Rah et al. reported that the reproducibility, fading effect, and angular dependency of RPLGD with a $6 \mathrm{MV}$ photon beam were $0.9 \%, 1.7 \%$, and $1.0 \%$, respectively $[19,20]$. All patient plans were delivered on an anthropomorphic RANDO female phantom (The Phantom Laboratory, Salem, NY) including two breasts $(10.8 \mathrm{~cm}$ in diameter and $4.3 \mathrm{~cm}$ in height) attached to a bottom plate, and organ doses were collected with detectors placed in the approximate middle of each organ. A total dose of 10 Gy was delivered due to limited sensitivity of RPLGD to scattered radiation. The average dose measured by the detectors was considered the organ equivalent dose (OED). Figure 2 displays detector positions in out-of-field organs in the female RANDO phantom, including the numbers of RPLGDs in parentheses.

\section{In-field organ doses based on dose-volume histograms}

Dosimetry for in-field organs (ipsilateral and contralateral lung, ipsilateral or contralateral heart, and contralateral breast) was based on the DVHs of each plan. The homogeneity index (HI), coverage index (CVI), and conformity index (CI) of the PTV and doses to lungs and heart were compared by treatment modality [21]. HI represents dose uniformity, defined as $H I=(|D 2-D 98| /$ $R x) \times 100$, where $D 2$ and $D 98$ are the doses received by $2 \%$ and $98 \%$, respectively, of the PTV in the DVH and $\mathrm{Rx}$ is the prescription dose [22]. By definition, smaller values of $\mathrm{HI}$ give better dose homogeneity in the PTV.
CVI is defined as $C V I=V 100 / V_{P T V}$, where $V 100$ is a volume receiving $100 \%$ of the prescription dose, and $V_{P T V}$ is a volume of PTV [22]. CI is defined as $C I=\left(V 95_{P T V} \times\right.$ $\left.V 95_{P T V}\right) /\left(V_{P T V} \times V 95\right)$, where $V 95_{P T V}$ is the PTV volume receiving $95 \%$ of the prescription dose, and $V 95$ is the body volume receiving $95 \%$ of the prescription dose [23]. In addition, the ipsilateral lung volumes receiving 20 Gy $\left(\mathrm{V} 20_{\mathrm{Gy}}\right)$ and $10 \mathrm{~Gy}\left(\mathrm{~V} 10_{\mathrm{Gy}}\right)$ are considered important parameters for evaluating the probability of pneumonitis [24-28].

We used the plateau dose-response model to estimate an OED for normal organs as follows [29, 30].

$$
O E D=\frac{1}{V} \sum_{i} V_{i}\left(\frac{1-\exp \left(-\delta D_{i}\right)}{\delta}\right)
$$

where $V$ is a body volume, and $V_{i}$ is a volume element exposed to a dose element $D_{i}$ [31]. In this model, a parameter $\delta$ was used to determine a dose-response curve for a specific organ. The OED values were used to estimate the LAR to each organ.

\section{Estimation of lifetime attributable risk to radiation dose}

As in the BEIR VII: Health Risks from Exposure to Low Levels of Ionizing Radiation report [32], the LAR for a person exposed to a radiation dose $(D)$ at age $(e)$ is expressed as follows:

$\operatorname{LAR}(D, e)=\int_{e+L}^{100}\left(E R R(D, e, a) \cdot \lambda_{l}^{C}\right)^{w} \times(E A R(D, e, a))^{1-w} \frac{s(a)}{s(e)} d a$, (2)

where ERR and EAR are an excess relative risk and an excess absolute risk, respectively, attained at the age of $a$ as a result of the radiation exposure at the age of $e . \lambda_{I}^{C}$ is the baseline cancer incidence rate and $w$ is the weight. $\mathrm{S}(a) / \mathrm{S}(e)$ is a ratio of the probability of surviving at the ages of $a$ and $e$, and $L$ is the latent period ( 5 years) for solid tumors. A weight (w) of 0.7 is encouraged by the BEIR Committee for most organs, although exceptions include breasts and lungs. For breasts, only the EAR model is recommended, and for lungs, a weight of 0.3 is recommended. The EAR and ERR model of BEIR VII are functions of sex $(x)$, age at exposure $(e)$, and attained age (a) as defined by the following equation: $\operatorname{LAR}(D, e)=\int_{e+L}^{100}\left(E R R(D, e, a) \cdot \lambda_{l}^{C}\right)^{w} \times(E A R(D, e, a))^{1-w} \frac{S(a)}{S(e)} d a$, (3)

where $\beta, \gamma$, and $\eta$ are model parameters for the age of exposure $(e)$ and the attained age $(a)$. Table 2 shows the parameters for preferred risk models in the BEIR VII report. In this study, we summed the age from $e+L$ to 100 years in LAR calculations for consistency with data from International Commission on Radiological Protection (ICRP) Report 103 [33]. 
Table 2: Parameters for preferred risk models in the BEIR VII: Health Risks from Exposure to Low Levels of Ionizing Radiation report.

\begin{tabular}{|c|c|c|c|c|c|c|c|c|}
\hline \multirow{2}{*}{ Site } & \multicolumn{4}{|c|}{ ERR model } & \multicolumn{4}{|c|}{ EAR Model } \\
\hline & $\boldsymbol{\beta}_{\mathrm{M}}$ & $\beta_{F}$ & $\gamma$ & $\eta$ & $\beta_{M}$ & $\beta_{F}$ & $\gamma$ & $\eta$ \\
\hline Lung & 0.32 & 1.4 & -0.3 & -1.4 & 2.3 & 3.4 & -0.41 & 5.2 \\
\hline Breast $(a<50)$ & \multirow{2}{*}{\multicolumn{4}{|c|}{ Not used }} & & 9.4 & -0.51 & 3.5 \\
\hline Breast ( $(\mathrm{a}>=50)$ ) & & & & & & 9.4 & -0.51 & 1.1 \\
\hline Thyroid & 0.53 & 1.05 & -0.83 & 0 & \multicolumn{4}{|c|}{ Not used } \\
\hline Liver & 0.32 & 0.32 & -0.3 & -1.4 & 2.2 & 1 & -0.41 & 4.1 \\
\hline Stomach & 0.21 & 0.48 & -0.3 & -1.4 & 4.9 & 4.9 & -0.41 & 2.8 \\
\hline Colon & 0.63 & 0.43 & -0.3 & -1.4 & 3.2 & 1.6 & -0.41 & 2.8 \\
\hline Gonad & 0.27 & 0.45 & -0.3 & -2.8 & 6.2 & 4.8 & -0.41 & 2.8 \\
\hline Rectum & 0.63 & 0.43 & $\mid-0.3$ & -1.4 & 3.2 & 1.6 & -0.41 & 2.8 \\
\hline
\end{tabular}

\section{RESULTS AND DISCUSSION}

\section{Comparison of the organ equivalent doses of the five treatment modalities}

Plan statistics for our 10 patients generated using the five treatment modalities are tabulated in Table 3. While the TOMO plans produced better dose homogeneity in the PTV, the IMRT and VMAT plans produced better PTV dose coverage and dose conformity. The V20Gy of the ipsilateral lung was the lowest in the IMRT plan, followed by the VMAT, 3D-CRT, TOMO, and FinF plans. However, The V10Gy was the highest for the VMAT plan among the five modalities. Therefore the IMRT plan produced the least probability of radiation-induced pneumonitis.

As shown in Figure 3, the OEDs in in-field organs were the highest with the VMAT plans. Most in-field OEDs were the lowest with the IMRT plans, but the outof-field OEDs for IMRT were higher than those for the 3D-CRT, FinF, and TOMO plans. The average number of MUs of the IMRT plans was 7 times that of the 3D-CRT plans. This large MU value was caused by the high modulation of MLCs. This high modulation with IMRT possibly caused the higher dose for out-of-field OARs such as the colon, gonad, and rectum as shown as Table 3.

Even though the VMAT plan used lower MUs as compared to the IMRT plan, the VMAT plans resulted in higher OEDs to the majority of the critical structures due to the higher volume of irradiation. This tendency is similar to the results of Lee et al. [6]. We found that the TOMO plans offered the lowest OEDs in most of the outof-field organs, while its in-field OEDs were comparable to those of the IMRT plan. Figure 4 shows an example of the DVHs for the PTV, contralateral breast, heart, ipsilateral lung, and contralateral lung of a representative patient. These results show that the plan quality of the
TOMO plans was better or comparable to that of the IMRT plans.

\section{Comparison of lifetime attributable risk of the five treatment modalities}

Table 4 shows for each modality the estimated LAR (based on calculations or measurements) to the organs of a secondary malignancy for an exposure at age between 30 and 100 years. As shown in Figure 5, LAR for each organ depends on its distance from the primary beam and the used modality. Therefore, higher LARs were obtained in the ipsilateral lung, thyroid, contralateral lung, and contralateral breast, in that order. In in-field organs, the LARs were the highest for the VMAT plans, followed by the IMRT plans. The higher LARs in the out-of-field organs for the IMRT and VMAT plans are attributed to large MUs and larger volume of irradiation [34]. The values of LAR of the TOMO plans were comparable to or lower than those of the 3D-CRT and FinF plans. For the LAR of all of the organs totaled, the TOMO plans had a value of $2083 \pm 255$ among 100,000 persons, which is the lowest value compared with the other modalities. The LAR of all organs for the VMAT plan was twice that of the TOMO plans. Therefore, the VMAT plans had especially high risk relative to the others.

An interesting finding was that the TOMO plans produced the lowest maximum and the highest mean dose ( $95 \%$ of the prescription dose) to the skin of the ipsilateral breast, while the IMRT plans provided the maximum skin dose (Table 5). This advantage for patients treated with TOMO can eliminate the use of a bolus to maintain good dose coverage while minimizing adverse effects on skin.

Some studies have reported that uncertainty in outof-field dose calculation by treatment planning systems is approximately $50 \%$ where the region of isodose is less than $10 \%$ of the prescription dose $[35,36]$. Therefore, the dose uncertainty in DVHs of the out-of-field organs might 
(a)

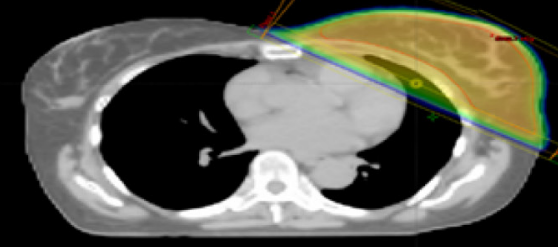

(c)

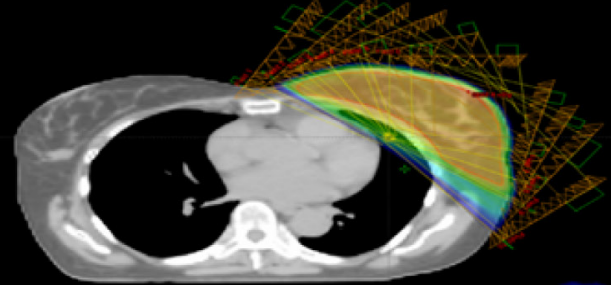

(e)

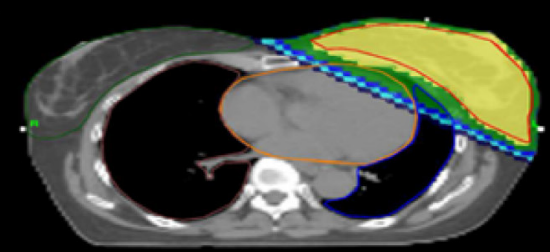

(b)

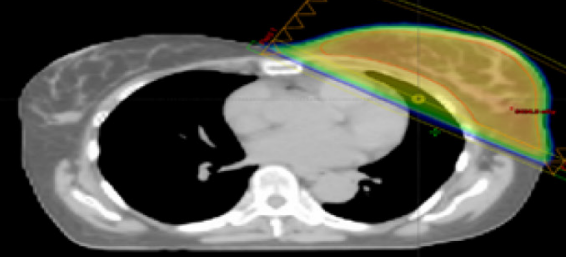

(d)

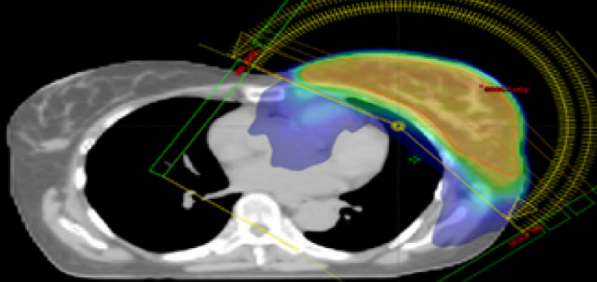

Figure 1: Graphical view of the isodose lines in the axial view for the patient 1 corresponding to Table 1 for the a. threedimensional conformal radiation therapy (3D-CRT) plan, b. field-in-field forward-planned intensity-modulated radiation (FinF) plan, c. intensity-modulated radiation therapy (IMRT) plan, d. volumetric modulated arc therapy (VMAT) plan, and e. TOMO plan.

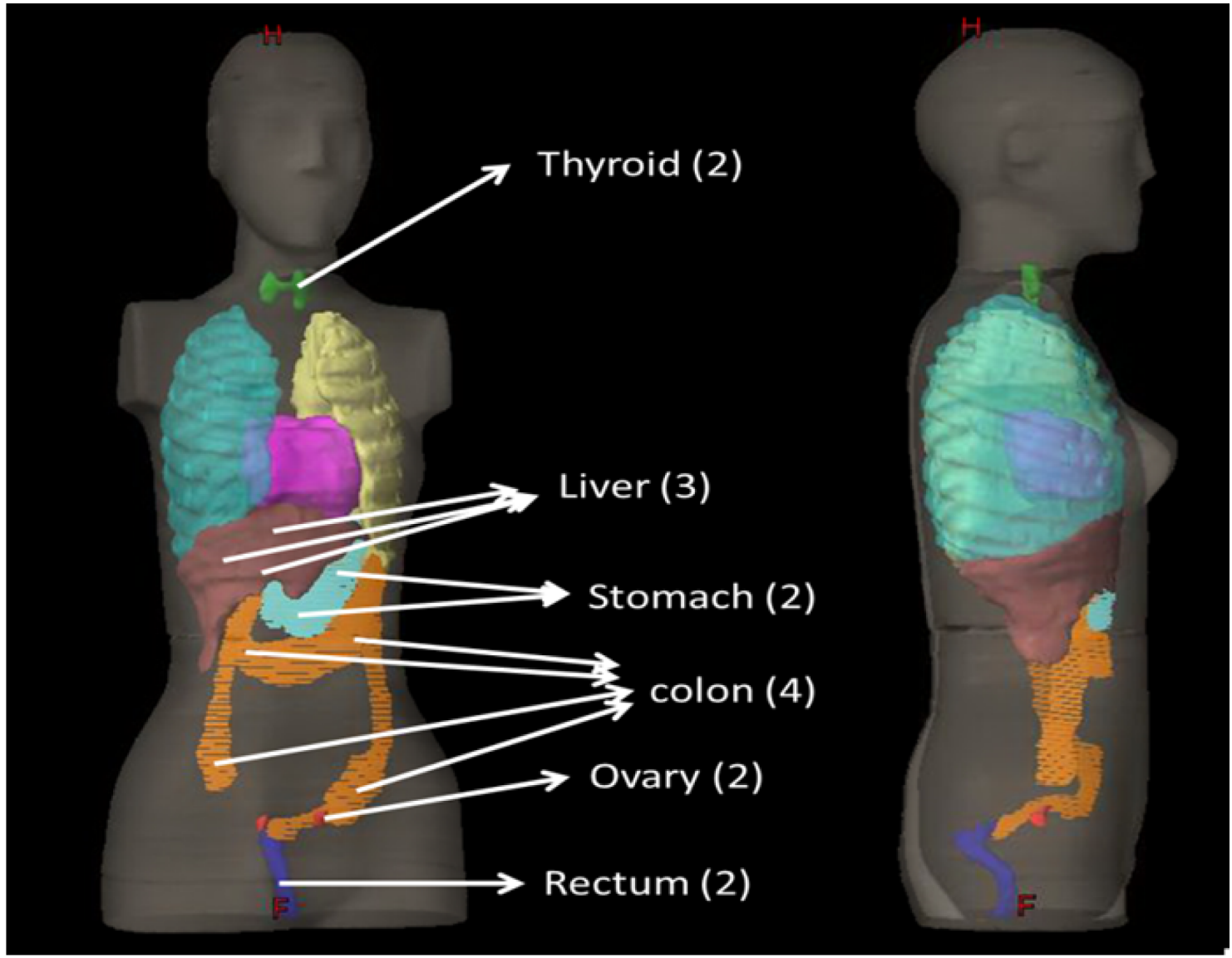

Figure 2: Detector positions in out-of-field organs (with numbers of RPLGDs in parentheses) in the female RANDO phantom. 


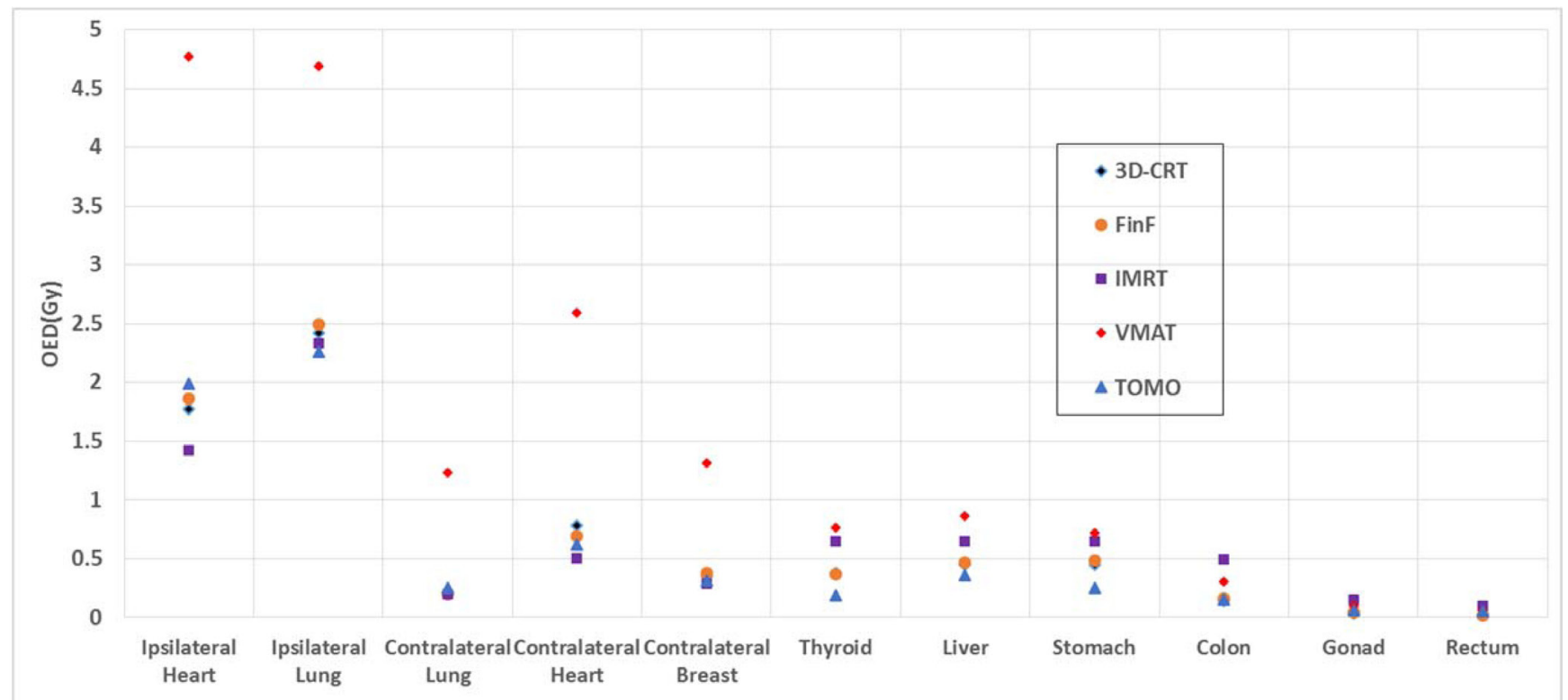

Figure 3: OEDs of in-field and out-of-field organs for the five treatment modalities.
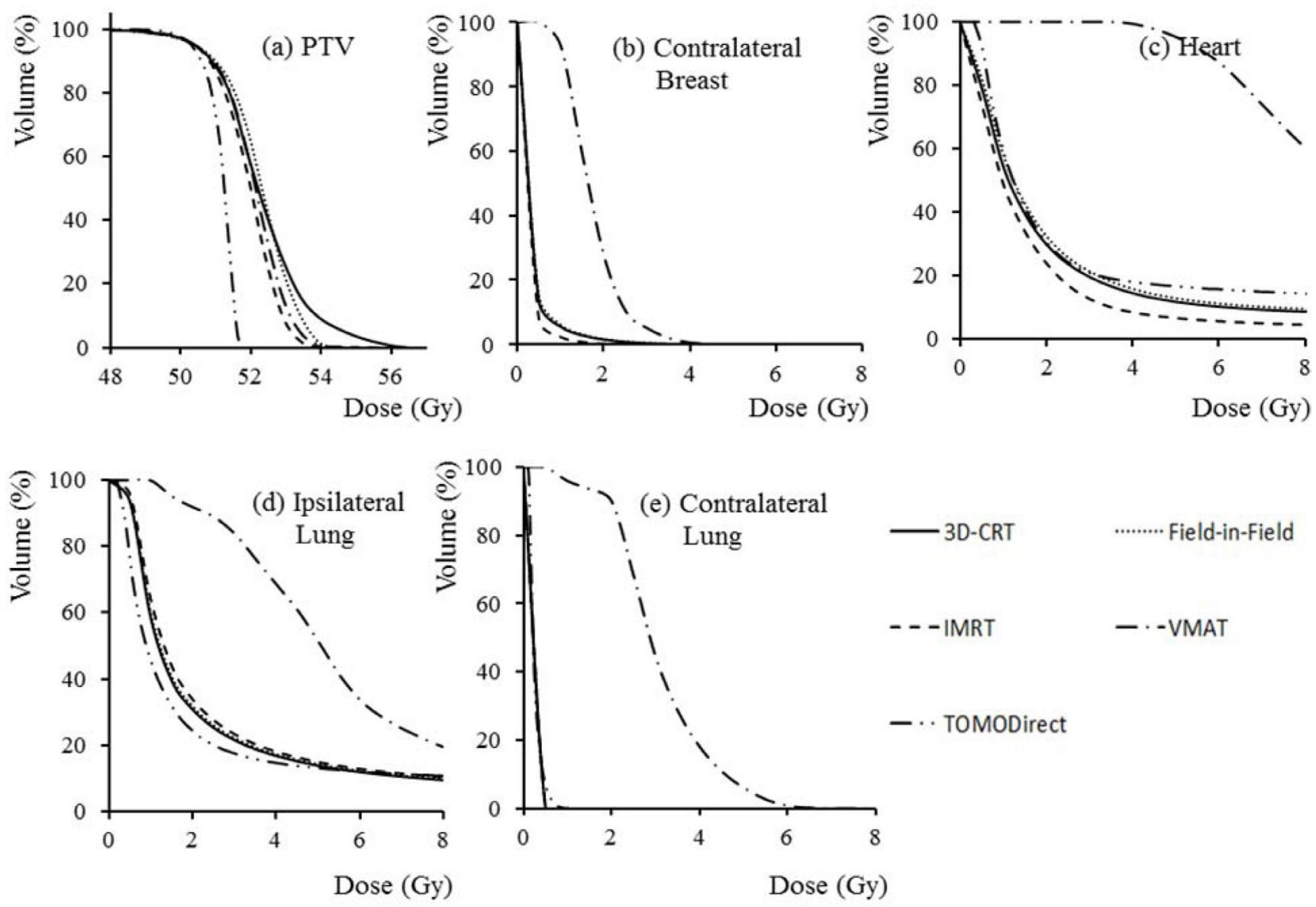

Figure 4: The dose-volume histograms for the a. planning target volume, b. contralateral breast, c. heart, d. ipsilateral lung, and e. contralateral lung of one patient for the five treatment modalities. 
Table 3: Patient plan information and findings (mean \pm standard deviation) for the five modalities.

\begin{tabular}{|c|c|c|c|c|c|c|c|}
\hline Region & Site & Item & 3D-CRT & FinF & IMRT & VMAT & TOMO \\
\hline & \multicolumn{2}{|c|}{ No. of fields/arcs } & 2 & 2 & 10 to 12 & 3 to 4 & 2 to 4 \\
\hline & \multicolumn{2}{|c|}{$\begin{array}{l}\text { Monitor units per } \\
\text { fraction }\end{array}$} & $229 \pm 13$ & $220 \pm 11$ & $1479 \pm 271$ & $460 \pm 51$ & $2807 \pm 655$ \\
\hline \multirow{10}{*}{ In field } & \multirow{3}{*}{ PTV coverage } & $\mathrm{HI}$ & $13.02 \pm 1.78$ & $11.74 \pm 2.29$ & $10.62 \pm 2.18$ & $11.41 \pm 1.83$ & $6.67 \pm 2.81$ \\
\hline & & CVI & $1.44 \pm 0.18$ & $1.41 \pm 0.25$ & $1.13 \pm 0.06$ & $1.10 \pm 0.08$ & $1.34 \pm 0.16$ \\
\hline & & $\mathrm{CI}$ & $0.60 \pm 0.09$ & $0.62 \pm 0.10$ & $0.82 \pm 0.03$ & $0.83 \pm 0.04$ & $0.51 \pm 0.08$ \\
\hline & \multirow{2}{*}{ Ipsilateral lung } & $\mathrm{V} 10^{\mathrm{a}}$ & $16.88 \pm 5.65$ & $17.50 \pm 6.82$ & $11.66 \pm 4.73$ & $29.32 \pm 10.02$ & $17.18 \pm 6.19$ \\
\hline & & $\mathrm{V} 20^{\mathrm{a}}$ & $12.93 \pm 5.15$ & $13.48 \pm 6.27$ & $6.97 \pm 3.59$ & $8.98 \pm 5.17$ & $13.42 \pm 5.68$ \\
\hline & Ipsilateral lung & $\mathrm{OED}^{\mathrm{b}}$ & $2.42 \pm 0.44$ & $2.49 \pm 0.43$ & $2.33 \pm 0.64$ & $4.69 \pm 0.87$ & $2.26 \pm 0.40$ \\
\hline & $\begin{array}{l}\text { Contralateral } \\
\text { lung }\end{array}$ & OED & $0.21 \pm 0.12$ & $0.20 \pm 0.10$ & $0.20 \pm 0.10$ & $1.23 \pm 1.05$ & $0.25 \pm 0.07$ \\
\hline & Ipsilateral heart & OED & $1.77 \pm 0.62$ & $1.86 \pm 0.63$ & $1.42 \pm 0.52$ & $4.77 \pm 0.75$ & $1.99 \pm 0.65$ \\
\hline & $\begin{array}{l}\text { Contralateral } \\
\text { heart }\end{array}$ & OED & $0.78 \pm 0.58$ & $0.69 \pm 0.21$ & $0.50 \pm 0.08$ & $2.59 \pm 1.11$ & $0.62 \pm 0.13$ \\
\hline & $\begin{array}{l}\text { Contralateral } \\
\text { breast }\end{array}$ & OED & $0.35 \pm 0.13$ & $0.38 \pm 0.14$ & $0.29 \pm 0.18$ & $1.31 \pm 0.55$ & $0.31 \pm 0.07$ \\
\hline \multirow{6}{*}{$\begin{array}{l}\text { Out of } \\
\text { field }\end{array}$} & Thyroid & OED & $0.38 \pm 0.13$ & $0.37 \pm 0.14$ & $0.65 \pm 0.21$ & $0.76 \pm 0.43$ & $0.19 \pm 0.04$ \\
\hline & Liver & OED & $0.45 \pm 0.17$ & $0.47 \pm 0.16$ & $0.65 \pm 0.13$ & $0.86 \pm 0.39$ & $0.36 \pm 0.10$ \\
\hline & Stomach & OED & $0.45 \pm 0.21$ & $0.48 \pm 0.20$ & $0.65 \pm 0.13$ & $0.72 \pm 0.18$ & $0.25 \pm 0.07$ \\
\hline & Colon & OED & $0.14 \pm 0.03$ & $0.16 \pm 0.09$ & $0.49 \pm 0.13$ & $0.30 \pm 0.10$ & $0.15 \pm 0.02$ \\
\hline & Gonad & OED & $0.03 \pm 0.01$ & $0.04 \pm 0.01$ & $0.15 \pm 0.05$ & $0.10 \pm 0.02$ & $0.06 \pm 0.01$ \\
\hline & Rectum & OED & $0.02 \pm 0.01$ & $0.02 \pm 0.01$ & $0.10 \pm 0.03$ & $0.06 \pm 0.01$ & $0.05 \pm 0.01$ \\
\hline
\end{tabular}

${ }^{a}$ Percentage of treatment volume that received $10 \mathrm{~Gy}$ or $20 \mathrm{~Gy} .{ }^{\mathrm{b}}$ Organ equivalent dose (in Gy).

Table 4: The lifetime attributable risk (LAR) of secondary malignancy in the organs at exposure summed to 100 years (mean \pm standard deviation).

\begin{tabular}{|l|l|l|l|l|l|}
\hline \multirow{2}{*}{ Site } & \multicolumn{4}{|l|}{ Lifetime attributable risk (among 100,000 population) } \\
\cline { 2 - 6 } & 3D-CRT & FinF & IMRT & VMAT & TOMO \\
\hline Ipsilateral lung & $1474 \pm 270$ & $1511 \pm 263$ & $1408 \pm 390$ & $2851 \pm 532$ & $1374 \pm 244$ \\
\hline Contralateral lung & $129 \pm 73$ & $120 \pm 61$ & $134 \pm 53$ & $746 \pm 639$ & $154 \pm 40$ \\
\hline Contralateral breast & $95 \pm 34$ & $104 \pm 38$ & $83 \pm 46$ & $355 \pm 148$ & $83 \pm 19$ \\
\hline Thyroid & $506 \pm 176$ & $496 \pm 192$ & $868 \pm 280$ & $1013 \pm 573$ & $255 \pm 55$ \\
\hline Liver & $42 \pm 16$ & $44 \pm 15$ & $60 \pm 12$ & $79 \pm 36$ & $33 \pm 10$ \\
\hline Stomach & $100 \pm 47$ & $107 \pm 45$ & $146 \pm 29$ & $162 \pm 40$ & $55 \pm 15$ \\
\hline Colon & $85 \pm 51$ & $94 \pm 55$ & $237 \pm 80$ & $181 \pm 60$ & $87 \pm 12$ \\
\hline Gonad & $6 \pm 3$ & $7 \pm 3$ & $29 \pm 10$ & $19 \pm 4$ & $12 \pm 1$ \\
\hline Rectum & $11 \pm 4$ & $14 \pm 4$ & $62 \pm 21$ & $36 \pm 5$ & $30 \pm 5$ \\
\hline Total & $2448 \pm 340$ & $2497 \pm 341$ & $2965 \pm 493$ & $5442 \pm 1024$ & $2083 \pm 255$ \\
\hline
\end{tabular}

Table 5: Skin doses (Gy) of five treatment modalities.

\begin{tabular}{|l|l|l|l|l|l|}
\hline & 3D-CRT & FinF & IMRT & VMAT & TOMO \\
\hline Minimum Dose & $18.5 \pm 6.4$ & $19.6 \pm 4.4$ & $11.1 \pm 3.9$ & $11.3 \pm 2.8$ & $23.6 \pm 7.7$ \\
\hline Maximum Dose & $56.3 \pm 1.3$ & $55.0 \pm 1.0$ & $57.2 \pm 1.1$ & $54.6 \pm 1.5$ & $53.4 \pm 1.1$ \\
\hline Mean Dose & $43.7 \pm 1.9$ & $43.2 \pm 1.4$ & $41.6 \pm 2.4$ & $39.4 \pm 2.3$ & $47.9 \pm 1.1$ \\
\hline
\end{tabular}



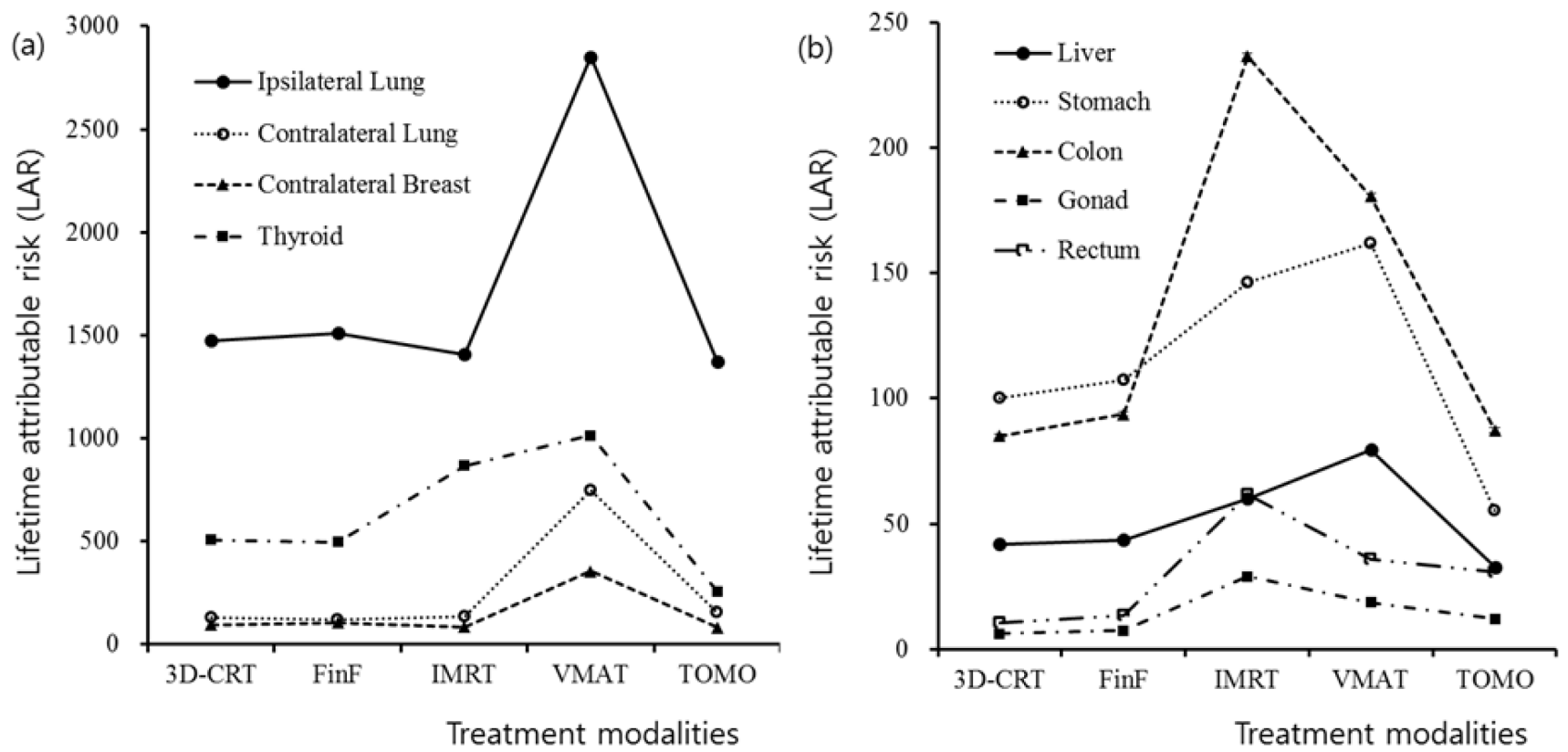

Figure 5: The lifetime attributable risk of secondary malignancy in each organ at risk for the five treatment modalities. a. Ipsilateral lung, contralateral lung, contralateral breast, and thyroid. b. Liver, stomach, colon, gonad, and rectum.

not be small. In our measurements, standard deviations in dose to the small organs such as thyroid and gonad, where detectors were located close to each other, were $6.2 \%$ and $4.9 \%$, respectively. In larger organs, the uncertainty increased with larger distance between the detectors.

Even though the risk of radiogenic cancer is generally proportional to the absorbed dose, there are nonnegligible uncertainties in the risk model including doseresponse relationship for carcinogenesis and modeling parameters. The latest report on radiation risk suggested that decisive choices among the several dose-response models are not possible based on the empirical data [32]. Therefore, there may be large inherent uncertainties in the risk estimation.

An increase in LAR is attributed to the increase in leakage as a result of increased MUs from a traditional Linac. However, with the TOMO plan, the MUs are much greater than those of other modalities, yet the summed LAR was the lowest among modalities. Because while $\mathrm{X}$-ray source of traditional Linac rotates around a patient continuously or at multiple static angles while the patient is lying still in a stationary couch, the TomoHDA system delivers the beam in a slice-by-slice fashion and each slice is collimated by the jaw in a moving couch, the definition of MU differs inherently for this modality. Therefore, MUs of TOMO cannot be explicitly compared to MUs of a traditional Linac.

The low summed LAR of the TOMO plan is mainly the result of two to three tangential IMRT fields delivered by a narrow field $(5.0 \mathrm{~cm})$. Therefore, our result might be limited to whole-breast radiation therapy. However, others have reported that the TomoHDA system provides better shielding $[35,37]$ and sharper dose gradient due to shorter source-to-skin distance $(85 \mathrm{~cm})$ and the narrower field width than those of the traditional Linac. These factors might have helped to further reduce the systemic LAR.

\section{CONCLUSIONS}

This comparative study shows that even though an IMRT plan provides overall better plan quality and the lowest probability of pneumonitis, it causes the second highest total LAR, after a VMAT plan. Our results indicate that a TOMO plan provides a plan quality comparable to an IMRT plan and poses the lowest risk of LAR to infield organs such as the ipsilateral lung, and to out-offield organs. Therefore, TOMO can be a better treatment modality for younger patients who have a longer life expectancy.

\section{ACKNOWLEDGMENTS}

This work was supported by the General Researcher Program (NRF-2015R1D1A1A09056828) and the Nuclear Safety Research Program (Grant No. 1403019) through the Korea Foundation, of Nuclear Safety (KOFONS), granted financial resource from the Nuclear Safety and Security Commission (NSSC), Republic of Korea.

\section{CONFLICTS OF INTEREST}

The authors declare no conflicts of interest. 


\section{REFERENCES}

1. Bartelink $\mathrm{H}$, Horiot $\mathrm{J}$ and Poortmans P. Recurrence rates after treatment of breast cancer with standard radiotherapy with or without additional radiation. N Eng J Med. 2001; 345:1378-1387.

2. Early Breast Cancer Trialists' Collaborative Group. Effect of radiotherapy after breast-conserving surgery on 10year recurrence and 15-year breast cancer death: metaanalysis of individual patient data for 10801 women in 17 randomized trials. Lancet. 2011; 12:1707-1716.

3. Boice J, Harvey E, Blettner M, Stovall M and Flannery J. Cancer in the contralateral breast after radiotherapy for breast cancer. N Engl J Med. 1992; 326:781-785.

4. Early Breast Cancer Trialists' Collaborative Group. Favorable and unfavorable effects on long term survival of radiotherapy for early breast cancer: an overview of the randomized trials. Lancet. 2000; 355:1757-1770.

5. Donovan E, James H, Bonora M, Yarnold J and Evans $\mathrm{P}$. Second cancer incidence risk estimates using BEIR VII models for standard and complex external beam radiotherapy for early breast cancer. Medical physics. 2012; 39:5814-5824.

6. Lee B, Lee S, Sung J and Yoon M. Radiotherapy-induced secondary cancer risk for breast cancer: 3D conformal therapy versus IMRT versus VMAT. Journal of radiological protection 2014; 34:325-331.

7. Preston D, Mattsson A, Holmberg E, Shore R, Hildreth $\mathrm{N}$ and Boice JJ. Radiation effects on breast cancer risk: a pooled analysis of eight cohorts. Radiation research. 2002; 158:220-235.

8. Yin Y, Chen J, Sun T, Ma C, Lu J, Liu T and Wang R. Dosimetric research on intensity-modulated arc radiotherapy planning for left breast cancer after breastpreservation surgery. Medical Dosimetry. 2012; 37:287292.

9. Abo-Madyan Y, Aziz M, Aly, MM, Schneider F, Sperk E, Clausen S, Giordano F, Hersking C, Steil V, Wenz F and Glatting G. Second cancer risk after 3D-CRT, IMRT and VMAT for breast cancer. Radiother Oncol. 2014; 110:471476.

10. Van Gestel D, van Viliet-Vroegindeweig C, Van den Heuvel F, Crijns W, Coelmont A, De Ost B, Holt A, Lamers E, Geussens Y, Nuyts S, Van den Weyngaert D, Van den Wyngaert T, Vermorken JB, Gregoire V. RapidArc, SmartArc and TomoHD compared with classical step and shoot and sliding window intensity modulated radiotherapy in an oropharyngeal cancer treatment plan comparison. Radiat Oncol. 2013; 8:1-11.

11. Emami B LJ, Brown A, Coia L, Goitein M, Munzenrider JE, Shank B, Solin LJ, Wesson M. Tolerance of normal tissue to therapeutic irradiation. Int J Radiat Oncolo Biol Phys. 1991; 21:109-122.

12. Li R XL, Horst K, Bush K. Nonisocentric treatment strategy for breast radiation therapy: A proof of concept study. Int $\mathrm{J}$ Radiat Oncol Biol Phys. 2014; 88:920-926.

13. Howell R, Scarboro S, Kry S and Yaldo D. Accuracy of out-of-field dose calculations by a commercial treatment planning system. Phys Med Biol. 2010; 55:6999-7008.

14. Schneider U, Hälg R, Hartmann M, Mack A, Storelli F, Joosten A, Möckli R and Besserer J. Accuracy of outof-field dose calculation of tomotherapy and cyberknife treatment planning systems: a dosimetric study. Z Med Phys. 2014; 24:211-215.

15. Arakia F, Moribe N, Shimonobou T and Yamashita Y. Dosimetric properties of radiophotoluminescent glass rod detector in high-energy photon beams from a linear accelerator and cyber-knife. Medical Physics. 2004; 31:1980-1986.

16. Hsu S, Yeh S, Lin M and Chen W. Comparison on characteristics of radiophotoluminescent glass dosemeters and thermoluminescent dosemeters. Radiation protection dosimetry. 2006; 119:327-331.

17. H Huang D and Hsu S, Radio-photoluminescence glass dosimeter (RPGLD). In: Gali-Muhtasib H, ed. Advances in Cancer Therapy (Intech, 2011). Available from: http:// www.intechopen.com/books/advances-in-cancer-therapy/ radio-photoluminescence-glass-dosimeter-rplgd-.

18. Nanto H, Takei Y and Miyamoto Y. (2011). Environmental Background Radiation Monitoring utilizing passive solid state dosimeters. (Intech, 2011).

19. Rah J HJ, Kim G, Kim Y, Shin D, Suh T. A comparison of the dosimetric characteristics of a glass rod dosimeter and a thermoluminescent dosimeter for mailed dosimeter. Radiation Measurements. 2008; 44:18-22.

20. Chung WK KD. Characteristic study of a radiophotoluminescence glass rod detector for clinical usages: Skin and inner body in-vivo verification. J Kor Phys Soc. 2013; 62:670-676.

21. Feuvret L, Noel G, Mazeron J and Bey P. Conformity index: a review Int J Radiat Oncol Biol Phys. 2006; 64:333342.

22. Wu Q, Mohan R, Morris M, Lauve A and Schmidt-Ullrich R. Simultaneous integrated boost intensity-modulated radiotherapy for locally advanced head-and-neck squamous cell carcinomas. I: dosimetric results Int J Radiat Oncol Biol Phys. 2003; 56:573-585.

23. Paddick I. A simple scoring ratio to index the conformity of radiosurgical treatment plans. Technical note. Journal of neurosurgery. 2000; 99:219-222.

24. Willner J, Jost A, Baier K and Flentje M. A little to a lot or a lot to a little? An analysis of pneumonitis risk from dose-volume histogram parameters of the lung in patients with lung cancer treated with 3-D conformal radiotherapy. Strahlentherapie und Onkologie. 2003; 179:548-556.

25. Roeder F, Friedrich J, Timke C, Kappes J, Huber P, Krempien R, Debus J and Bischof M. Correlation of patientrelated factors and dose-volume histogram parameters with 
the onset of radiation pneumonitis in patients with small cell lung cancer. Strahlentherapie und Onkologie. 2010; 186:149-156.

26. Yilmaz S, Adas Y, Hicsonmez A, Andrieu M, Akyurek $\mathrm{S}$ and Gokce S. Evaluation of the radiation pneumonia development risk in lung cancer cases. Asian Pacific journal of cancer prevention. APJCP. 2014; 15:7371-7375.

27. Dang J, Li G, Zang S, Zhang S and Yao L. Risk and predictors for early radiation pneumonitis in patients with stage III non-small cell lung cancer treated with concurrent or sequential chemoradiotherapy. Radiation oncology. 2014; 9:172.

28. Blom G, Wennberg B, Svane G, Bylund H and Lind P. Reduction of radiation pneumonitis by V20-constraints in breast cancer. Radiation oncology. 2010; 5:99.

29. Hall EJ WC. Radiation-induced second cancers: the impact of 3D-CRT and IMRT Int J Radiat Oncol Biol Phys. 2003; 56:83-88.

30. Davis R. Production and killing of second cancer precursor cells in radiation therapy: in regard to Hall and Wuu. Int J Radiat Oncol Biol Phys. 2004; 59:916.

31. Schneider U and Kaser-Hotz B. Radiation risk estimates after radiotherapy: application of the organ equivalent dose concept to plateau dose-response relationships Radiation and environmental biophysics. 2005; 44:235-239.
32. National Research Council of the National Academies. Health risks from exposure to low levels of ionizing radaition, BEIR VII Phase 2. 2006 (Washington DC: The national Academic Press).

33. ICRP. The 2007 Recommendations of the International Commission on Radiological Protection. Publication 103. 2007:1-332.

34. Kim D, Chung K, Chung W, Bae S, Shin D, Hong S and al. e. Risk of secondary cancers from scattered radiation during intensity-modulated radiotherapies for hepatocellular carcinoma. Radiation oncology. 2014; 9:109.

35. Ramsey C, Seibert R, Mahan S, Desai D and Chase D. Outof-field dosimetry measurements for a helical tomotherapy system. J Appl Clin Med Phys. 2006; 7:1-11.

36. Howell R, Scarboro S, Taddei P, Krishnan S, Kry S and Newhauser W. Methodology for determining doses to infield, out-of-field and partially in-field organs for late effects studies in photon radiotherapy. Phys Med Biol. 2010; 55:7009-7023.

37. Bennett BR LM, Elson HR. Analysis of peripheral doses for base of tongue treatment by linear accelerator and helical TomoTherapy IMRT. J Appl Clin Med Phys. 2010; 11:3136. 